\title{
Luminosity Evolution of Double Radio Sources
}

\author{
Joel C. Carvalho ${ }^{1}$ and Christopher P. O’'Dea ${ }^{2}$ \\ ${ }^{1}$ Departamento de Fisica, UFRN, C.P. 1661, CEP 59072-970, Natal, RN, Brazil \\ carvalho@dfte.ufrn.br \\ ${ }^{2}$ Space Telescope Science Institute, 3700 San Martin Dr., Baltimore, MD 21218, USA \\ odea@stsci.edu
}

Received 2002 July 1, accepted 2003 January 16

\begin{abstract}
We have recently developed a detailed analytical model for powerful radio sources based on the results of a series of 2-D numerical hydrodynamical simulations. Here we make use of the model results to investigate the radio source luminosity evolution. Changes in the radio spectrum due to radiation losses were calculated in two different scenarios for energy gains/losses: Kardashev-Pacholczyk (KP) and 'continuous injection' (CI). The magnetic field was calculated in two limiting cases: (1) assuming equipartition of energy between relativistic particles and fields and (2) magnetic flux conservation inside the cocoon. The effect of the surrounding medium was taken into account by considering three different ambient density profiles. The evolutionary tracks were plotted in a power-diameter (P-D) diagram and compared with the predictions of self-similar models. In general, the evolutionary tracks cannot be represented by a simple power law and have a complex form that is most probably the result of the nonself-similar evolution of the source.
\end{abstract}

Keywords: galaxies: active — galaxies: jets — radio continuum: galaxies

\section{Introduction}

The power-diameter diagram is an important tool for probing how radio luminosity and radio source size vary over radio source lifetimes (Baldwin 1982; Fanti et al. 1995; Readhead et al. 1996; Kaiser et al. 1997; O’Dea \& Baum 1997; Blundell, Rawlings \& Willott 1999; Alexander 2000; Snellen et al. 2000).

We have recently developed a detailed analytical model for powerful radio sources (Carvalho \& O'Dea 2003, Paper III) based on the results of a series of numerical hydrodynamical simulations (Carvalho \& O'Dea 2002a,b). The model takes into account the nonuniform distribution of pressure in the shocked ambient gas region enclosed by the bow shock and cocoon and is in good agreement with the numerical simulations regarding the time dependence of the source volume, the variation of pressure with source size and the overall shape and aspect ratio of the cocoon and bow shock. In this paper we make use of the model results to investigate the radio source luminosity evolution. The radio luminosity of the source can be estimated if we make the simplified assumption that the energy density of the synchrotron particles is roughly equal to the gas hydrodynamic pressure.

We consider two limiting cases for the magnetic field: (1) equipartition of energy between relativistic particles and fields and (2) magnetic flux conservation inside the cocoon. Radiation losses are taken into account and two different models (Kardashev-Pacholczyk (KP) and 'continuous injection' (CI)) are used to calculate the change in the radio spectrum. We investigate the effect of the ambient gas by considering a constant density atmosphere, an isothermal atmosphere with a density gradient according to King's law and, finally, an atmosphere where the gas density follows King's law up to a distance
Table 1. Types of self-similar models

\begin{tabular}{llll}
\hline & Type I & Type II & Type III \\
\hline Density, $\rho$ & Constant & $d^{-2}$ & $d^{-\delta}$ \\
Head speed, $v_{\mathrm{h}}$ & Constant & Constant & $t^{(\delta-2) /(5-\delta)}$ \\
Dead area, $A_{\mathrm{h}}$ & Constant & $t^{2}$ & $t^{(\delta+4) /(5-\delta)}$ \\
Source size, $z_{\mathrm{h}}$ & $t$ & $t$ & $t^{(3) /(5-\delta)}$ \\
Head pressure, $P_{\mathrm{h}}$ & Constant & $t^{-2}$ & $t^{-(\delta+4) /(5-\delta)}$ \\
Cocoon radius, $r_{\mathrm{c}}$ & $t^{1 / 2}$ & $t^{1 / 2}$ & $t^{(3) /(5-\delta)}$ \\
Cocoon pressure, $P_{\mathrm{c}}$ & $t^{-1}$ & $t^{-1}$ & $t^{-(\delta+4) /(5-\delta)}$ \\
$P_{\mathrm{h} / P_{\mathrm{c}}}$ & $t$ & $t^{-1}$ & Constant \\
\hline
\end{tabular}

Type I: Begelman \& Cioffi (1989); Loken et al. (1992); Cioffi \& Blondin (1992); Nath (1995).

Type II: Daly (1990).

Type III: Falle (1991); Begelman (1996); Bicknell, Dopita \& O’Dea (1997); Kaiser \& Alexander (1997).

of $100 \mathrm{kpc}$ from the galactic nuclei and then remains constant.

We also study the luminosity evolution within the framework of three different self-similar models (see Table 1) for the source expansion assuming that the bulk of the radio emission comes either from the source head or from the whole cocoon. In this instance, the radio emissivity is assumed to be uniform over the emitting region. We use our more detailed analytical model to calculate the evolutionary tracks for sources generated by jets with a broad range of physical parameters.

\section{Results and Discussion}

We compare our results with the predictions of the selfsimilar models in Table 2 which gives values for the exponent $d$, where $P_{178} \propto z_{\mathrm{h}}^{d}$, for both the head and the 
Table 2. Evolution of radio power - exponent $d$ in $P_{v} \propto z_{\mathrm{h}}^{d}$

\begin{tabular}{lcccccc}
\hline Emitting volume & \multicolumn{2}{c}{ Constant density } & & \multicolumn{3}{c}{ Decaying density } \\
\cline { 2 - 3 } \cline { 6 - 7 } & \multicolumn{2}{c}{ Model type } & & \multicolumn{2}{c}{ Model type } \\
& I $\quad$ III $(\delta=0)$ & & II & III $(\delta=3 / 2)$ & III $(\delta=2)$ \\
\hline Head & 0 & $-1 / 3$ & & $-1 / 2$ & $-1 / 2$ & $-11 / 24$ \\
Cocoon & $1 / 4$ & $2 / 3$ & & $1 / 4$ & $-1 / 2$ & $-5 / 24$ \\
$\quad$ Equipartition field & $1 / 4$ & $-1 / 3$ & & 1 & $-1 / 2$ & $-11 / 24$ \\
$\quad$ Flux conservation field & $-1 / 2$ & & &
\end{tabular}
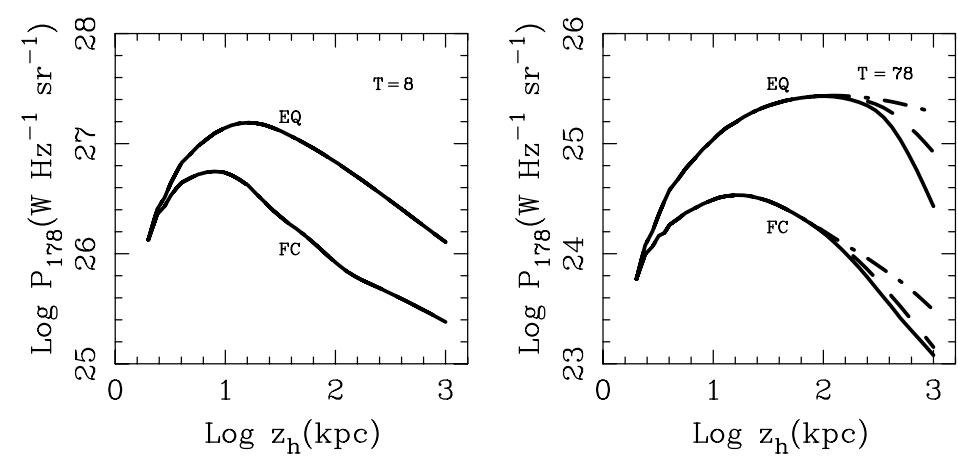

Figure 1 Comparison between different energy gain/loss processes. The left panel is for a young source $(M=38.8, \eta=0.0542)$ and the right panel for an old source $(M=3.3, \eta=0.021)$. Full lines represent the $\mathrm{KP}$ model, dashed lines the CI model and dashed-dotted lines no energy loss. Equipartition magnetic field is indicated by 'EQ' and flux conservation field by 'FC'. Here, $M$ is the Mach number, $\eta$ is the jet to ambient density ratio and $z_{\mathrm{h}}$ is the source size. The source age $T$ is in Myr and the redshift $z=1$.

cocoon volume. We see that the different self-similar models predict significantly different values.

A comparison of the effects of radiative losses (Fig. 1) shows that in the young source, the effects of radiative losses are negligible, while in the older source, the results can be very significant. The KP loss model results in lower radio powers, especially in sources with equipartition magnetic fields. The increase in inverse Compton losses going from $z=1$ to $z=2$ causes a significant reduction in radio power.

In a constant density atmosphere, the radio luminosity of our analytical model tends to increase with time (size). An exception is the model with flux conserving magnetic field which is roughly constant or slightly decreasing in luminosity. The effect of radiative losses is more dramatic at low and intermediate Mach number since these sources are older than those with comparable size but with high $M$. In the case of an equipartition field the exponent $d$ tends asymptotically to approximately $-1 / 3$ after a start at $d \sim 2 / 3$. This is more apparent for the KP model.

In an atmosphere with declining density, the radio luminosity initially rises, but then declines with increasing size. The source size for which the maximum occurs decreases with increasing Mach number. The radio powersize relation does not follow a simple power law although at intermediate sizes it agrees approximately with the prediction of Type III self-similar models.

For a given jet power, cocoons with flux conserving magnetic field have lower radio luminosity than cocoons with equipartition magnetic fields. In constant density atmospheres, cocoons with magnetic flux conservation dim faster than those with equipartition fields.

In an atmosphere with declining density, sources dominated by a pair plasma (electron + positron jet) experience relatively lower radiative losses and have higher radio power than sources dominated by baryons (electron + proton jet).

We have also considered an atmosphere where the gas density follows King's law up to a distance of 100 $\mathrm{kpc}$ from the galactic nucleus and then remains constant (Fig. 2). In the regions where the IGM density is constant, the radio power decreases more slowly than the case where the density continues to decline. For both the equipartition field and flux conservation field, the radio power, after initially increasing, falls with source size roughly as $z_{\mathrm{h}}^{-1 / 2}$, except in the case of low Mach number for the KP model. Coincidentally, this is the behavior predicted by Type III self-similar models which predict $d=-1 / 2$ and $d=-1 / 3$ for $z_{\mathrm{h}}<100 \mathrm{kpc}(\delta=2)$ and $z_{\mathrm{h}}>100 \mathrm{kpc}(\delta=0)$, respectively, at least in the case of a flux conserving field (even though the self-similar models do not include radiation losses).

Finally, we have shown (Carvalho \& O'Dea 2003) that in a detailed analytical model based on hydrodynamical simulations, the jet propagation does not exhibit self-similar behavior. Self-similar models will render a power law for the luminosity-size relation. However, the evolutionary tracks shown in Figure 2 cannot be 
Radio Power

Run R11 - R15

Case 1: electrons

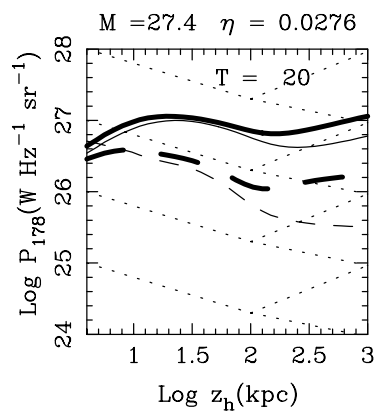

Radio Power

Run R16 - R20

Case 2: electrons + protons

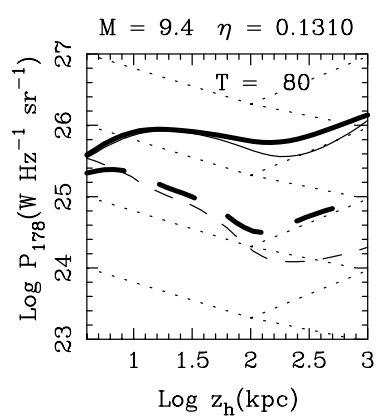

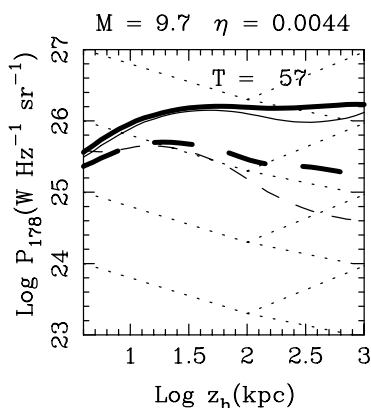
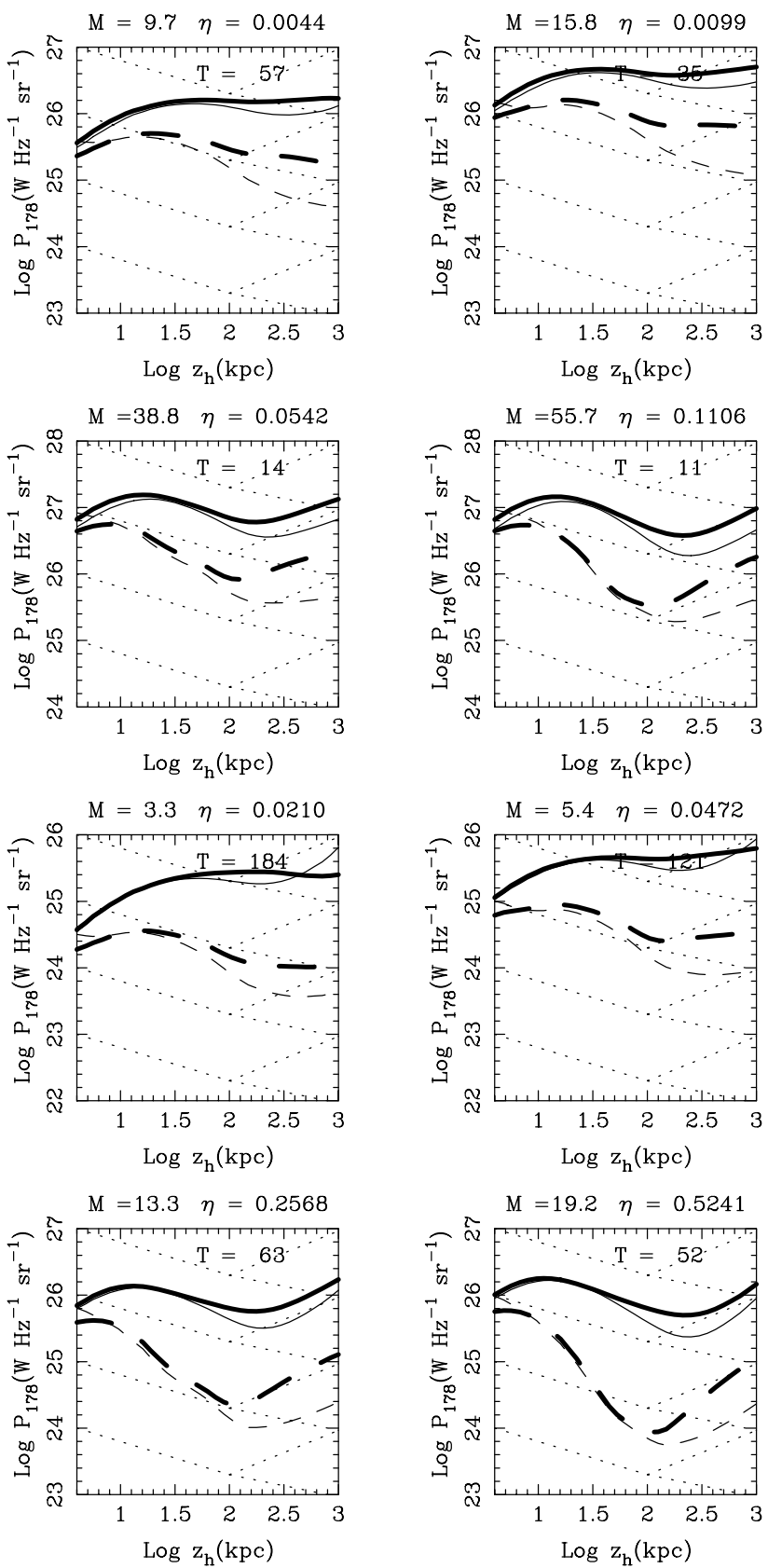

Figure 2 Radio power as a function of source size for jets propagating in an atmosphere where the gas density follows King's law up to a distance of $100 \mathrm{kpc}$ from the galactic nuclei and then remains constant, taking into account radiation losses with continuous injection (CI model) for a source redshift $z=1$. The dotted lines are the prediction of Type III self-similar models, that is, $d=-1 / 2$ in the case of an equipartition field and flux conserving field for $z_{\mathrm{h}}<100 \mathrm{kpc}$ and $d=2 / 3$ and $d=-1 / 3$ respectively for an equipartition and flux conserving field for $z_{\mathrm{h}}>100 \mathrm{kpc}$. The full lines are for an equipartition field and the dashed lines for flux conservation field. The thin lines represent the radio power calculated assuming uniform average energy density in the cocoon. The thick lines denote the radio power calculated integrating the emissivity along the cocoon length.

represented by a simple power law and instead have a complex form that is most probably the result of the nonself-similar evolution of the source.

\section{Acknowledgments}

JCC acknowledges the financial support of PRONEX/ Finep and CNPq and the hospitality of STScI where this work was carried out.

\section{References}

Alexander, P. 2000, MNRAS, 319, 8

Baldwin, J. E. 1982, In Extragalactic Radio Sources, eds

D. S. Heeschen \& C. M. Wade (Dordrecht: Reidel), 21

Begelman, M. C. 1996, in Cygnus A: Study of a Radio Galaxy, eds

C. L. Carilli \& D. A. Harris (Cambridge University Press), 209

Begelman, M. C., \& Cioffi, D. F. 1989, ApJ, 345, L21

Bicknell, G. V., Dopita, M. A., \& O’Dea, C. P. 1997, ApJ, 485, 112

Blundell, K. M., Rawlings, S., \& Willott, C. J. 1999, AJ, 117, 677

Carvalho, J. C., \& O'Dea, C. P. 2002a (Paper I), ApJS, 141, 337. 
Carvalho, J. C., \& O'Dea, C. P. 2002b (Paper II), ApJS, 141, 371. Carvalho, J. C., \& O’Dea, C. P. 2003 (Paper III), in preparation. Cioffi, D. F., \& Blondin, J. M. 1992, ApJ, 392, 458 Daly, R. A. 1990, ApJ, 355, 416

Falle, S. A. E. G. 1991, MNRAS, 250, 581

Fanti, C., Fanti, R., Dallacasa, D., Schilizzi, R. T., Spencer, R. E., \& Stanghellini, C. 1995, A\&A, 302, 317

Kaiser, C. R., \& Alexander, P. 1997, MNRAS, 286, 215

Kaiser, C. R., Dennett-Thorpe, J., \& Alexander, P. 1997, MNRAS, 292,723
Loken, C., Burns, J. O., Clarke, D. A., \& Norman, M. L. 1992, ApJ, 392, 54

Nath, B. B. 1995, MNRAS, 274, 208

O'Dea C. P., \& Baum S. A. 1997, AJ, 113, 148

Readhead A. C. S., Taylor G. B., Pearson T. J., \& Wilkinson P. N. 1996, ApJ, 460, 634

Snellen, I. A. G., Schilizzi, R. T., Miley, G. K., de Bruyn, A. G., \& Bremer, M. N. 2000, MNRAS, 319, 445 